\title{
Examining the acceptability of an online dietary recall tool among differing ethnic groups living in Ireland
}

\author{
G. Bennett ${ }^{1,2}$, S. Yang ${ }^{1,2}$, L.A. Bardon ${ }^{1,2}$ and E.R. Gibney ${ }^{1,2}$ \\ ${ }^{1}$ Institute of Food and Health, University College Dublin, Dublin, Ireland and \\ ${ }^{2}$ School of Agriculture and Food Science, University College Dublin, Dublin, Ireland
}

Over the past decade, substantial progress has been made in the development of novel dietary assessment technologies ${ }^{(1)}$. Advantages of online dietary assessment tools include cost-time effectiveness, greater participant reach and improved data quality ${ }^{(1)}$. Foodbook24 is an online 24-hour dietary recall tool designed for the Irish adult population ${ }^{(2)}$. As Ireland is becoming an increasingly diverse country $^{(3)}$, so too are foods consumed here. To accommodate assessment of diverse diets in Ireland the existing food list within Foodbook24 has been expanded to include 420 ethnic and 136 new Irish foods. Foodbook24(including food lists and questions) was translated into Mandarin, Polish and Portuguese to accommodate the use of the tool among Chinese, Polish and Brazilian groups. This work examines the acceptability and usability of the extended food lists.

To compare Foodbook24's recently expanded food list to common foods consumed in Irish, Chinese, Polish and Brazilian diets, participants completed a personal reflection of their habitual diet using a Pinterest Board, consisting of at least 20 food images(pins). A Pinterest Board template, divided into four sections; Breakfast, Lunch, Dinner, Snacks, was sent to participants to help them structure the foods they consume. Participants were asked to 'pin' images of foods commonly consumed at each meal. The completed Pinterest Boards were supported by a voice recording where participants explained how they prepare, cook and serve their food (stage 1). Two non-consecutive 24-hour dietary recalls via Foodbook24 and an evaluation questionnaire will assess dietary differences between ethnic groups and determine the feasibility of Foodbook24 among these groups(stage 2).

A total of $n=11$ have completed Stage 1 of the study. Of these, 100\% identify as Irish, 4 male and 7 female. The mean age is $40.5 \pm$ 18.8 years. All participants $(n=11)$ completed personal reflections using a guided methodology on Pinterest. The mean number of pins(foods) described at meals were as follows; breakfast $n=6$, lunch $n=6$, dinner $n=9$, snacks $n=9$.A total of $n=237$ foods were mentioned in the personal reflections, with $92.5 \%$ of these foods included in the expanded Foodbook 24 food list. Most foods omitted from Foodbook24's food list were oils and condiments. Of $n=11$ voice recordings, $n=6$ mentioned consuming different foods on weekends than weekdays. $\mathrm{N}=6$ participants serve meals as individual portions rather than in a communal dish. Recruitment for this study is ongoing. Basic descriptive statistics and content analysis will be performed on all data collected.

Preliminary analysis shows that the expanded food list within Foodbook 24 comprehensively covers the food items consumed by the adult Irish population. A lower variety of foods were consumed at breakfast and lunch times. Further analysis will identify if there are foods commonly consumed by Chinese, Polish and Brazilian people missing from the tool's food list and will assess the acceptability of Foodbook24 among four ethnic groups in Ireland.

\section{Acknowledgements}

Funding: This work is part of the Food Nutrition Security Cloud (FNS-Cloud) which has received funding from the European Union's Horizon 2020 Research and Innovation programme.

\section{References}

1. Amoutzopoulos, Steer, Roberts, et al. (2018) J Nutr Sci 7, e11.

2. Timon, Evans, Kehoe, et al. (2017) Nutrients, 9(5), 425.

3. Central Statistics Office (2016) Profile 7 Migration and Diversity. 